\title{
A Non-Neuronal Cholinergic System Regulates Cellular ATP Levels to Maintain Cell Viability
}

\author{
Shino Oikawa Mitsue Iketani Yoshihiko Kakinuma \\ Department of Physiology, Nippon Medical School Graduate School of Medicine, Tokyo, Japan
}

\section{Key Words}

A non-neuronal cholinergic system • Acetylcholine • Energy metabolism • Glucose uptake

\begin{abstract}
Background/Aims: We previously suggested that a non-neuronal cholinergic system modulates energy metabolism through the mitochondria. However, the mechanisms responsible for making this system crucial remained undetermined. Methods: In this study, we developed a fusion protein expression vector containing a luciferase gene fused to the folic acid receptor- $\alpha$ gene. Results: This protein of the vector was confirmed to target the plasma membrane of transfected HEK293 cells, and vector-derived luciferase activities and ATP levels in viable cells were positively correlated $(r=0.599)$. Using this luciferase vector, choline acetyltransferase (ChAT)-expressing cells (i.e., cells with an activated non-neuronal cholinergic system) had increased cellular ATP levels. ChAT-expressing cells also had upregulated IGF1R and Glut-1 protein expressions as well as increased glucose uptake. This activated nonneuronal cholinergic system with efficient glucose metabolism rendered cells resistant to serum depletion-induced cell death. Conclusion: Our results indicate that a non-neuronal cholinergic system is involved in sustaining ATP levels to render cells resistant to a nutrientdeficient environment.
\end{abstract}

Copyright (C) 2014 S. Karger AG, Basel

\section{Introduction}

We previously demonstrated that a non-neuronal cholinergic system in cells was involved in regulating cellular energy metabolism by negatively modulating mitochondrial function. This was observed by knockdown of a rate-limiting enzyme choline acetyltransferase (ChAT), responsible for acetylcholine (ACh) synthesis, leading to reciprocally accelerated oxygen

Yoshihiko Kakinuma, M.D., Ph.D. Graduate School Professor
Dep. of Physiology, Nippon Medical School Graduate School of Medicine 1-1-5 Sendagi, Bunkyo-ku, Tokyo 113-8602 (Japan)

Tel. +81-3-3822-2131 Ext 5244, Fax +81-3-3822-0766, E-Mail k12417853@nms.ac.jp 


\section{Cellular Physiology and Biochemistry}

Cell Physiol Biochem 2014;34:781-789

\begin{tabular}{l|l}
\hline DOI: $10.1159 / 000363042$ & (c) 2014 S. Karger AG, Basel
\end{tabular}

Oikawa/Iketani/Kakinuma: A non-Neuronal Cholinergic System that Regulates Cellular ATP Levels

consumption by knockout cells [1]. Based on this function, cells in which ChAT was deleted were susceptible to cell death, particularly under hypoxic conditions, produced more reactive oxygen species after treatment with norepinephrine, and had excessive reductions in cellular ATP levels [2]. These results suggested that a non-neuronal cholinergic system prevented mitochondria from excessive oxygen consumption or oxidative stress $[1,2]$.

We also recently developed transgenic mice with cardiac ventricle-specific overexpression of this system and found that the heart was spared from ischemic insults caused by accelerated angiogenesis and augmented cardiac glucose uptake through enhanced cardiomyocyte-expression of glucose transporter (Glut)-4. Consequently, the heart acquired ischemic or hypoxic resistance [3]. These results suggested that a non-neuronal cholinergic system could definitely sustain cellular ATP levels and strengthened the link between this system and energy metabolism. These beneficial results led us to speculate that this cellular non-neuronal cholinergic system was involved in efficient utilization of specific energy substrates and maintenance of cellular ATP levels.

However, a system for real-time monitoring of cellular ATP levels is still required, as opposed to the current conventional methods used along with commercially available kits, because in these conventional methods, ATP levels are evaluated using a cell-lysis procedure, and they cannot be continuously measured using identical cells. Cellular ATP quantification methods are based on direct measurements of ATP levels in cell-lysates, i.e., ATP levels in the same viable cells cannot be monitored or estimated in a sequential manner. Consequently, real-time monitoring of cellular ATP levels, particularly under pathological conditions, is impossible. However, advances in technology have gradually enabled monitoring ATP levels in viable cells.

Based on a previous study [4], we developed a fusion protein expression vector that comprised a luciferase gene flanked by the membrane-localization signals of the folic acid receptor- $\alpha$ gene. The benefit of using this vector was that by measuring luciferase activity in culture medium, cellular ATP levels could be monitored in real-time. Using this specific vector, we investigated our hypothesis that compared with ChAT non-expressing cells ChATexpressing cells may have increased ATP levels.

\section{Materials and Methods}

\section{Cell culture}

HEK293 cells were purchased from the Japanese Collection of Research Bioresources Cell Bank, National Institute of Biomedical Innovation (Osaka, Japan). They were cultured in Dulbecco's modified Eagle's medium (DMEM; Wako Pure Chemical Industries, Ltd., Osaka, Japan) with low glucose (1000 mg/L) and supplemented with 10\% FBS and antibiotics. HEK293 cells transfected with the expression vector pEBMulti-Hyg (Wako Pure Chemical Industries, Ltd.), harboring a gene of interest, were cultured in the presence of hygromycin $(500 \mu \mathrm{g} / \mathrm{mL})$ to obtain a stable transformant. In a serum starvation experiment, cells were treated with fresh DMEM without serum and were continuously cultured without changing the medium for more than 1 week until cell death was observed.

\section{Membrane-targeting luciferase expression vector}

With reference to a previous report [4], we constructed a membrane-targeting luciferase expression vector. The human folic acid receptor-gene was subcloned into a pIRES-EGFP expression vector and digested with two appropriate restriction enzymes, SalI and PstI, which left a part of each the $5^{\prime}$ and $3^{\prime}$ region of the gene in the expression vector being responsible for membrane targeting of the folic acid receptor. A fulllength luciferase gene that harbored additional SalI and PstI sites was inserted into the folic acid receptor- $\alpha$ gene that was already subcloned into the pIRES-EGFP vector. The luciferase gene was verified to be inserted in-frame to the folic acid receptor- $\alpha$ gene. This constructed expression vector was designated as the Luc-FR vector (Luc-FR). As previously reported [2], pEBMulti-Hyg (Wako Pure Chemical Industries, Ltd.) was used to overexpress the murine $C h A T$, the full coding sequence of which was subcloned into this vector and was designated as the ChAT vector (ChAT). To transiently express both Luc-FR and ChAT, the Effectene cationic 


\section{Cellular Physiology \\ and Biochemistry}

Cell Physiol Biochem 2014;34:781-789

\begin{tabular}{l|l}
\hline DOI: $10.1159 / 000363042$ & (c) 2014 S. Karger AG, Basel
\end{tabular}

Oikawa/Iketani/Kakinuma: A non-Neuronal Cholinergic System that Regulates Cellular ATP Levels

transfection reagent (QIAGEN, Hamburg, Germany) was used. After 48 h, transfected cells were used for experiments. Stably pEBMulti-Hyg vector-transfected cells or pIRES-EGFP vector-transfected cells were used as the negative control for transfection experiments.

\section{Immunocytochemical analysis}

Transfected cells were cultured on glass bottom dishes (Matsunami Glass Int., Ltd. Osaka, Japan), washed several times with PBS, fixed with $4 \%$ paraformaldehyde, and permeabilized with $0.1 \%$ Triton $\mathrm{X}-100$. After blocking with $0.1 \% \mathrm{BSA}$, cells were treated with a primary antibody at $4{ }^{\circ} \mathrm{C}$ overnight: either a goat anti-luciferase polyclonal antibody (1:100 dilution; Promega Co. Madison, WI, USA) or a goat anti-ChAT polyclonal antibody (1:100 dilution; Merck Millipore, Billerica, MA, USA). After reaction with an appropriate secondary antibody conjugated with a fluorescent dye, including Alexa Fluor 546 rabbit anti-goat IgG (Life Technologies, Tokyo, Japan), immunofluorescent signals were observed by confocal laser microscopy.

\section{Western blot analysis}

As in our previous studies $[1,5,6]$, Western blot analysis was performed using specific antibodies along with appropriate secondary antibodies. Antibodies against Glut-1 (Santa Cruz Biotechnology, Inc., Dallas, Texas, USA) and the IGF-1 receptor (IGF-1R; Cell Signaling Technology, Danvers, Massachusetts, USA) were used (each at 1:1000 dilution). Proteins were detected using an Immobilon Western Chemiluminescent HRP Substrate (Merck Millipore).

Luciferase assay

At 48-h after transfection with Luc-FR alone or with Luc-FR and ChAT, cells were treated with PBS containing 20- $\mu \mathrm{M}$ luciferin (WAKO Chemicals) at $37^{\circ} \mathrm{C}$ for $15 \mathrm{~min}$ in the presence or absence of ATP at different concentrations ( $1 \mathrm{nM}-1000 \mu \mathrm{M})$, followed by measurements of luciferase activities in PBS using a luminometer (GloMax ${ }^{\circledR}$ 20/20 Luminometer, Promega). Transfection efficiency for HEK293 cells was corrected by the activity of Sea Pansy using a Sea Pansy reporter vector, which was transfected with the above vector, according to the manufacturer's protocol (Toyo B-Net Co., Ltd., Tokyo, Japan).

\section{Cellular ATP measurements}

Cellular ATP levels were measured in cell-lysates using a commercial kit (Amerix, Tokushima, Japan). With reference to a standard luciferase activity curve deduced by the corresponding ATP concentration, the actual ATP concentration was determined.

Glucose uptake using 2-NBDG

The glucose analogue 2-NBDG (Life Technologies) was used to assess cellular glucose uptake. 2-NBDG $(100 \mu \mathrm{M})$ was added to the culture medium of a stable transformant of HEK293 cells that expressed ChAT or control transformant cells. After $15 \mathrm{~min}$, cells were sequentially monitored for fluorescent signals. At optimized time points, cells were fixed with 4\% PFA and observed under a confocal microscope.

MTT activity assay

As previously reported [2], cell viability was determined using a commercial kit (Cell Counting Kit-8, Dojindo Laboratories, Kumamoto, Japan).

\section{Statistical analysis}

Results are presented as means \pm standard errors of the mean. Results for two groups were compared using a non-parametric Student's t-test. A $P$-value of $<0.05$ was considered significant.

\section{Results}

The Luc-FR vector induces luciferase protein expression in the plasma membrane

First, Luc-FR vector-transfected cells were examined to determine whether the luciferase protein synthesized by these cells was expressed in the plasma membrane. As shown by western blot analysis (Fig. 1A), transiently Luc-FR-transfected HEK293 cells expressed the 
Oikawa/Iketani/Kakinuma: A non-Neuronal Cholinergic System that Regulates Cellular ATP Levels

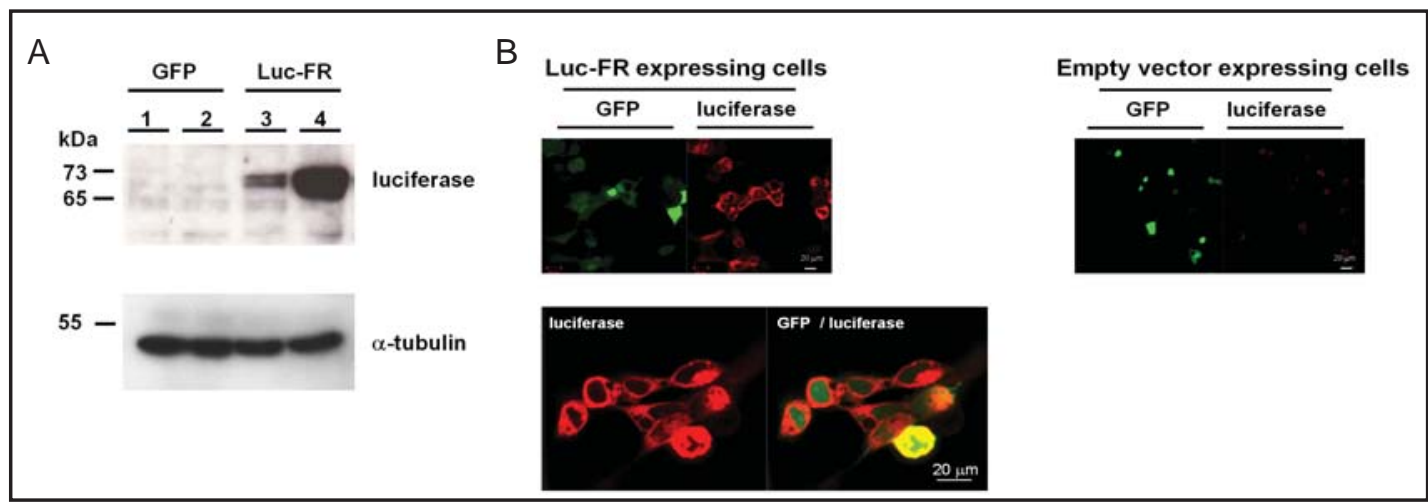

Fig. 1. Luciferase protein fused to a folic acid receptor $\alpha$ protein. A. HEK293 cells transiently transfected with the Luc-FR vector expressed the luciferase protein, as determined by Western blot analysis. Each lane represents a different cell type that either did (Luc-FR) or did not express luciferase (GFP). Representative data for three independent experiments are shown. B. Compared with an EGFP-expressing control vector, the luciferase protein clearly targeted the plasma membrane through the Luc-FR vector (red). Transfected cells were identified by EGFP-positive signals (green). Scale bars, $20 \mu \mathrm{m}$.

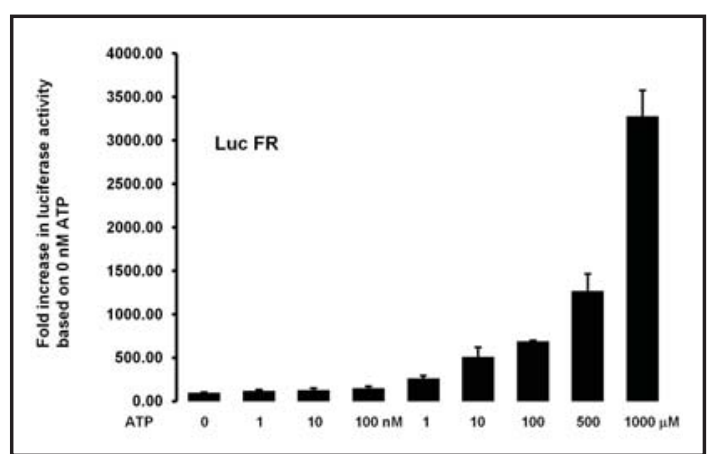

Fig. 2. Luciferase activity in PBS used for treating Luc-FR vector-transfected cells increased with an increasing extrinsic ATP threshold level. Compared with luciferase activity in PBS used for treating LucFR vector-transfected cells in the absence of ATP, there was no remarkable increase with extrinsic ATP levels between $1 \mathrm{nM}$ and $1 \mu \mathrm{M}(277.0 \pm 0.8 \%$, $\mathrm{n}$ $=6)$. However, luciferase activity abruptly increased with the addition of $>10-\mu \mathrm{M}$ extrinsic ATP, particularly $>500 \mu \mathrm{M}$ of ATP $(1266.3 \pm 196.2 \%, \mathrm{n}=6)$. Bars indicate average fold-increases in luciferase activity for 3-4 independent experiments.

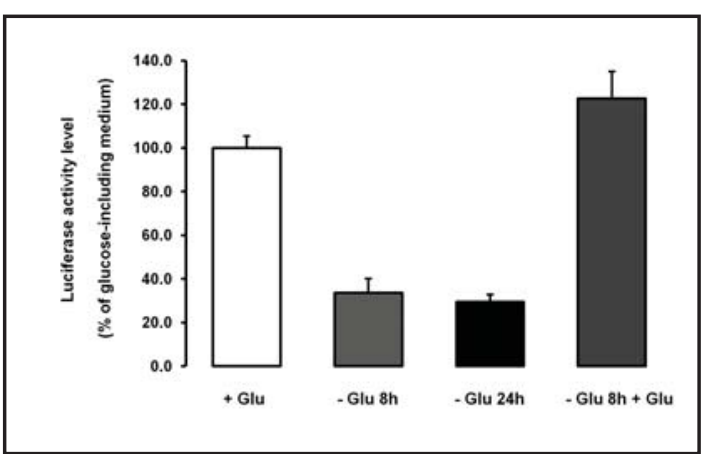

Fig. 3. Luc-FR vector-transfected cells sense the extracellular glucose level. Glucose deprivation alone reduced luciferase activity in PBS used for treating transfected cells within $8 \mathrm{~h}$ to $33.6 \pm 6.4 \%(P<0.01)$, followed by a further decrease to $29.4 \pm 3.4 \%(P<$ 0.01 ) within $24 \mathrm{~h}$; however, these cells remained alive. Luciferase activity that was downregulated with the $8 \mathrm{~h}$-glucose deprivation recovered and increased after adding glucose $(122.6 \pm 12.3 \%, P<0.01)$.

luciferase protein. Immunofluorescent analysis established that the luciferase protein had targeted the plasma membranes of these transfected cells, as shown by red band-like signals along these membranes (Fig. 1B).

The Luc-FR vector induces luciferase activity in cells in response to extrinsic ATP

In a previous study [4], membrane-targeted luciferase responded to ATP in the culture medium. Thus, we determined whether luciferase activity dose-dependently increased in response to the ATP concentration in the culture medium, which was added at final concentrations ranging from $1 \mathrm{nM}$ to $1000 \mu \mathrm{M}$. Luciferase activity slightly increased in the presence of 100-nM ATP $(151.6 \pm 21.0 \%, P<0.01, \mathrm{n}=3)$, and increased only minimally at a concentration of 1- $\mu$ M ATP (Fig. 2). However, luciferase activity significantly increased 
 \\ Oikawa/Iketani/Kakinuma: A non-Neuronal Cholinergic System that Regulates Cellular ATP Levels}

Fig. 4. The non-neuronal cholinergic system augmented by ChAT overexpression maintains increased cellular ATP levels. For ChATexpressing cells, the luciferase activity in PBS used for treating these cells was not upregulated after adding $100 \mu \mathrm{M}$ of ATP $(208.9 \pm$ $28.2 \%, P<0.01, \mathrm{n}=6$ ). However, there was an abrupt increase in luciferase activity after the addition of $>500 \mu \mathrm{M}$ of ATP $(1029.2$ $\pm 252.2 \%, P<0.01, \mathrm{n}=6-8$ ). This contrasted considerably with the results in Fig. 2. Bars indicate average fold-increases in luciferase activity for 3-4 independent experiments.

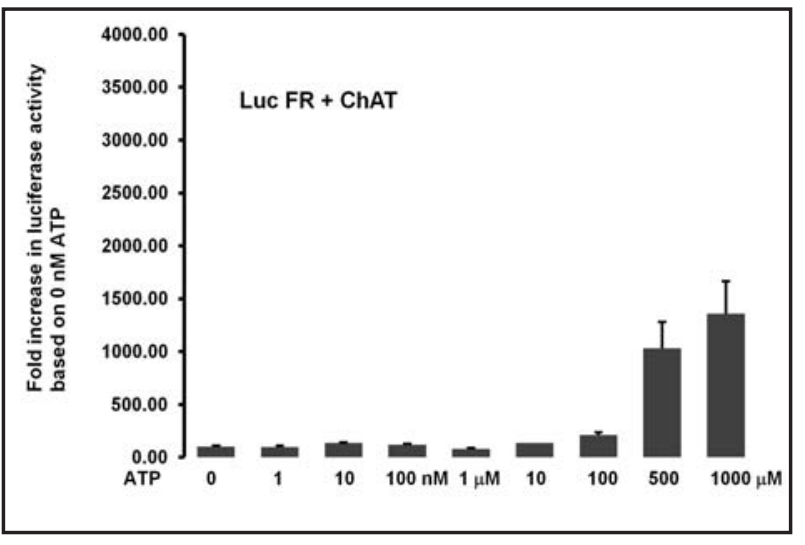

at a concentration of $>10-\mu \mathrm{M}$ ATP $(512.8 \pm 108.8 \%, P<0.01, \mathrm{n}=6)$. This suggested that luciferase activity did not respond to extrinsic ATP, when the intracellular ATP level exceeded the exogenous level, although it did increase after reaching a threshold that exceeded the intracellular ATP level.

\section{Effects of the culture medium glucose concentration on luciferase activity}

To assess the activity of the Luc-FR vector under certain pathological conditions, luciferase activity was determined in cells that were placed in a culture medium with a low glucose level (Fig. 3). Compared with the luciferase activity of control cells cultured with standard amounts of glucose $(100.0 \pm 5.6 \%)$, a glucose-free condition of $8 \mathrm{~h}$ drastically decreased the activity level to $33.6 \pm 6.4 \%(P<0.01, \mathrm{n}=9)$. However, cells remained alive during this time period and 24-h later, the activity level further decreased to $29.4 \pm 3.4 \%$ ( $P$ $<0.01, \mathrm{n}=6$ ). Glucose supplementation after this 8-h glucose depletion resulted in increased luciferase activity reaching its previous level under normal conditions $(122.6 \pm 12.3 \%, P<$ 0.01 vs. 8-h glucose free condition, $\mathrm{n}=9$ ).

Measurement of cellular ATP levels using the Luc-FR vector compared with those measured using a commercial kit

ATP concentrations in cells cultured in a 24-well plate were determined using a commercial ATP kit. Cellular ATP levels ranged from $10^{-6}$ to $10^{-5} \mathrm{M}$ with luciferase activities of approximately $10^{7}$. Prior cell lysis for ATP determination, luciferase activity in the PBS reaction mixture was determined in which Luc-FR vector-transfected cells were incubated (i.e., PBS supernatants used for luciferase activity measurements and cell lysates of the same cultured cells for cellular ATP measurements). With reference to a standard curve for cellular ATP concentrations, Luc-FR luciferase activity was also found to be approximately $10^{7}$, which was similar to the actual luciferase activity.

To validate the relationship between Luc-FR vector-derived luciferase activity and the actual cellular ATP level-dependent luciferase activity, we compared the relative increases in Luc-FR luciferase activity level (X, independent variable) with the actual ATP-dependent luciferase activity (dependent variable). The actual luciferase activity (X) and the actual ATP level $(\mathrm{Y})$ were described by the equation as follows: $\mathrm{Y}=5 \times 10^{6}+647405 \mathrm{X}$. The correlation coefficient ( $\mathrm{r}$ ) was 0.599291 and $\mathrm{R}^{2}=0.3592$. This suggested that the vector-derived luciferase activity positively correlated with the actual ATP-derived activity.

\section{ChAT overexpression effects on luciferase activity}

We previously reported that a non-neuronal cholinergic system is involved in suppressing cellular energy metabolism determined by knockdown of ChAT gene [1, 2]. However, whether cellular ATP levels were upregulated in ChAT-expressing cells remains undetermined. We used the Luc-FR vector to confirm an activation effect of a non-neuronal cholinergic system on ATP levels (Fig. 4). The fold-increases in luciferase activity in ChAT- 


\section{Cellular Physiology \\ Cell Physiol Biochem 2014;34:781-789 \\ and Biochemistry

\begin{tabular}{l|l}
\hline DOI: $10.1159 / 000363042$ & (C) 2014 S. Karger AG, Basel
\end{tabular} \\ Oikawa/Iketani/Kakinuma: A non-Neuronal Cholinergic System that Regulates Cellular ATP Levels}
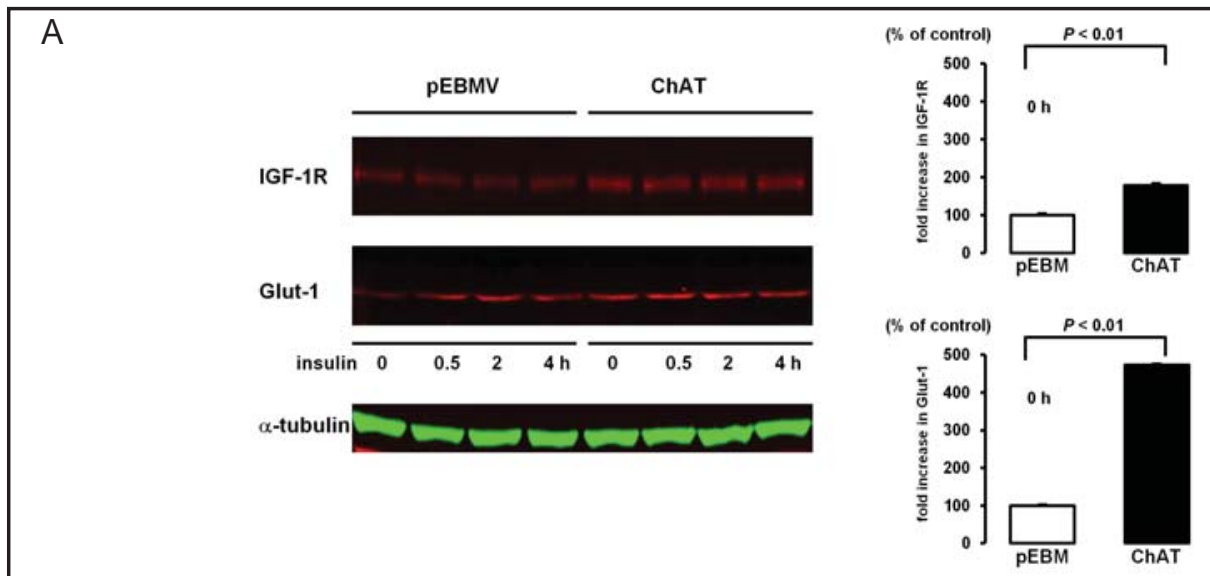

B

C
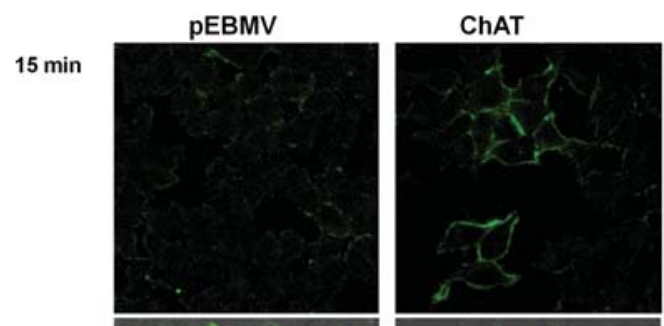

$90 \mathrm{~min}$
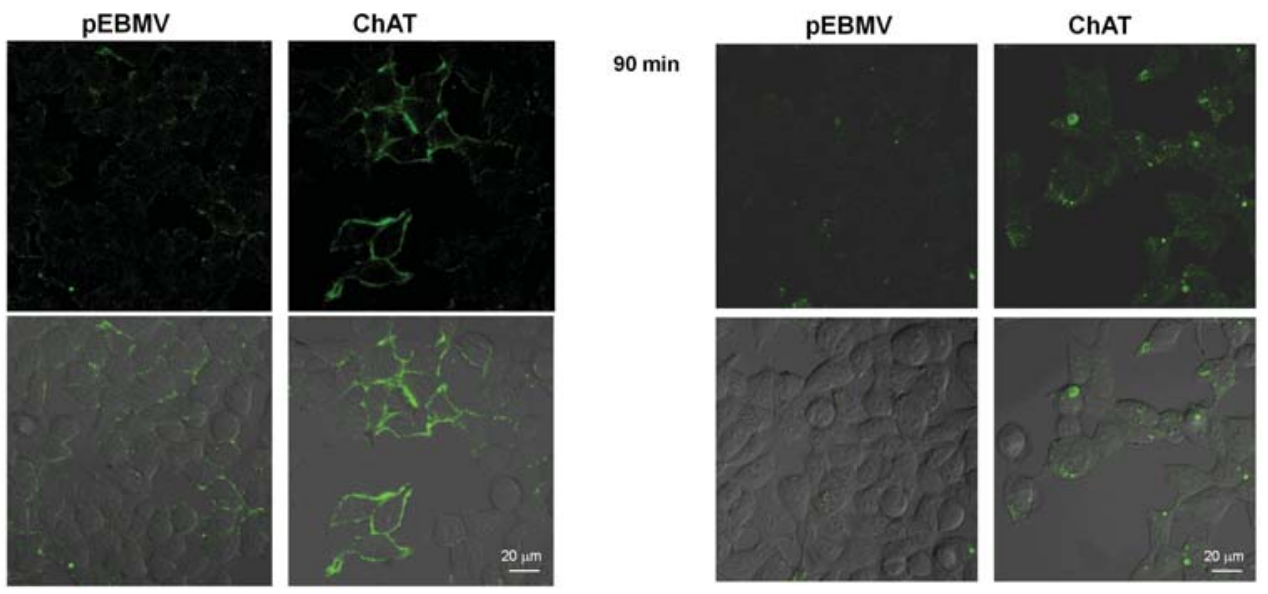

Fig. 5. Glucose is efficiently incorporated by ChAT-expressing cells. A. Western blot analysis showed that, even in the absence of insulin $(0.1 \mathrm{M})$, ChAT-expressing cells expressed more IGF-1R $(178.4 \pm 3.5 \%, P<0.01$ vs. control) and Glut-1 (473.0 $\pm 2.5 \%, P<0.01$ vs. control) proteins compared with ChAT non-expressing cells. B. Within $15 \mathrm{~min}$, ChAT-expressing cells rapidly incorporated 2-NDBG compared with control cells. In particular, strong 2-NDBG signals were observed along the plasma membrane of these cells. In contrast, weak signals were observed in the plasma membrane of control cells. C. After 90 min, more 2-NDBG signals appeared in ChAT-expressing cells and were observed predominantly in the cytoplasm, whereas few signals were observed in the cytoplasm of control cells. Representative data from 3-4 independent experiments are shown.

transfected cells sharply increased from $100 \mu \mathrm{M}$ to $500 \mu \mathrm{M}$ of ATP $(1029.2 \pm 252.2 \%, P<0.01$, $\mathrm{n}=6-8$; Fig. 4); however, it did not increase at concentrations of $<100 \mu \mathrm{M}$. This contrasted considerably with the results shown in Fig. 2 , in which luciferase activity was increased more by $10 \mu \mathrm{M}$ of ATP $(512.8 \pm 108.8 \%, P<0.01, \mathrm{n}=3-6)$. Because the activity response of ChATexpressing cells to exogenously-added ATP began after that of control cells, suggesting that ChAT was involved in sustaining ATP levels to a greater degree in these cells than in control cells. Using this Luc-FR vector system, ChAT-expressing cells was observed to have a higher baseline luciferase activity compared with control cells $(222.2 \pm 25.6 \%, P<0.01, \mathrm{n}=9)$, which supported our speculation noted above.

\section{ChAT overexpression induces IGF-1R and Glut-1 protein expression}

To investigate the phenotypes that resulted from the activation of this non-neuronal cholinergic system, we investigated the protein expression of IGF-1R, insulin receptor (IR), and Glut-1. Compared with control cells, ChAT-expressing cells already had increased 
 \\ Oikawa/Iketani/Kakinuma: A non-Neuronal Cholinergic System that Regulates Cellular ATP Levels}

Fig. 6. ChAT-expressing cells are resistant to serum deprivation. Serum deprivation for 1 week resulted in cell death in control cells. In contrast, ChAT-expressing cells were resistant to serum deprivation and cells that survived were three times more than control cells, as determined by the MTT activity assay (ChAT1: $328.1 \pm 44.4 \%$ and ChAT2: $389.7 \pm 94.8 \%, P<0.01)$.

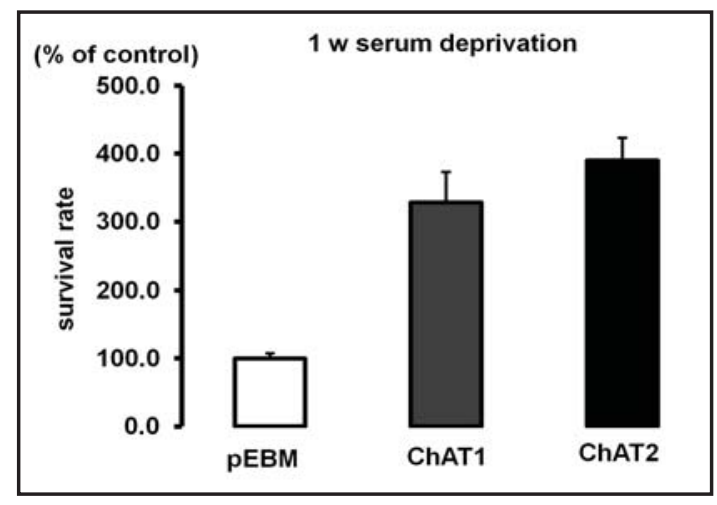

expressions of IGF-1R $(178.4 \pm 3.5 \%$ vs. control, $P<0.01, \mathrm{n}=4-5)$ and Glut- $1(473.0 \pm 2.5 \%, P$ $<0.01$ vs. control, $\mathrm{n}=4-5$ ) (Fig. $5 \mathrm{~A}$ ); both these proteins are involved in glucose uptake. With insulin $(0.1 \mu \mathrm{M})$, the Glut-1 response was augmented in ChAT-expressing cells. However, IR protein expression levels were comparable in transfected and control cells (data not shown). These results suggested that the upregulated non-neuronal cholinergic system resulted in increased glucose utilization.

\section{Enhanced glucose incorporation in ChAT-expressing cells}

To clearly demonstrate the role of this system, we performed a glucose incorporation assay using the glucose molecular probe 2-NDBG (Fig. 5B). Compared with control cells, ChAT-expressing cells efficiently incorporated 2-NDBG within 15-90 min (Fig. 5B and 5C). Within 15 min, ChAT-expressing cells more rapidly incorporated 2-NDBG than control cells. As a result, compared with control cells, more and stronger green 2-NDBG signals appeared in ChAT-expressing cells along their plasma membranes in a linear pattern. After $90 \mathrm{~min}$, more 2-NDBG was incorporated in ChAT-expressing cells and higher intensity signals were detected in their cytoplasm. These results indicated that this non-neuronal cholinergic system had increased glucose uptake.

\section{ChAT-expressing cell viability under serum deprivation condition}

Based on the results described above, this non-neuronal cholinergic system possibly had a beneficial role in cell survival by energy saving and efficient glucose utilization. Thus, we investigated the pathophysiological significance of this system. ChAT-expressing cells were subjected to continuous serum deprivation for 7 days. During this period, morphologies of control cells gradually appeared round and some of these cells died. In contrast, a larger number of ChAT-expressing cells survived during serum deprivation (ChAT1: $328.1 \pm 44.4 \%$ and ChAT2: $389.7 \pm 94.8 \%, P<0.01$ vs. control, $\mathrm{n}=8$; Fig. 6 ). These results suggested that this non-neuronal cholinergic system had a critical role in salvaging cells under poor nutrientdeficient environmental conditions probably through its energy-saving effects.

\section{Discussion}

We previously focused on a functional role of a non-neuronal cholinergic system [1]. Based on the results of the previous study, we found some novel effects of this system in which cells had increased ATP levels if this system was activated. However, we had not yet investigated the detailed effects of this system, because cellular ATP levels were only measured at a specific time point using a commercial ATP kit $[7,8]$. Thus, a more technically advanced method was required to monitor ATP levels in real-time. In this study, we developed a specific luciferase reporter vector with reference to a previous study [4], which enabled us to quantify cellular ATP levels using a culture medium supernatant without the need for cell lysis. 


\section{Cellular Physiology and Biochemistry}

Cell Physiol Biochem 2014;34:781-789

\begin{tabular}{l|l}
\hline DOI: $10.1159 / 000363042$ & C 2014 S. Karger AG, Basel
\end{tabular}

www.karger.com/cpb

Oikawa/Iketani/Kakinuma: A non-Neuronal Cholinergic System that Regulates Cellular ATP Levels

In the current study, we first showed that this reporter vector system was feasible for evaluating cellular ATP levels in real-time without the need for cell lysis. The fold-increases in luciferase activity observed in cells transfected with this vector were well correlated with the actual luciferase activity, which reflected the ATP levels in viable cells. When cellular ATP levels exceeded the extracellular ATP level, which was altered by adding ATP to the culture medium, luciferase reporter activity was not affected and showed no abrupt increases (Fig. 2). However, when the extrinsic ATP level exceeded cellular ATP levels, luciferase activity was affected by this extrinsic ATP and increased exponentially relative to the extrinsic level. The fold-increases in luciferase activity in control cells exceeded $500 \%$ after adding $>10 \mu \mathrm{M}$ of ATP (Fig. 2). However, introducing ChAT shifted the extrinsic ATP concentration required for a $>500 \%$ increase in the luciferase activity to $100 \mu \mathrm{M}-500 \mu \mathrm{M}$ (Fig. 4). This indicated that ChAT-expressing cells contained more ATP than ChAT non-expressing cells. Thus, the usage of this novel reporter vector system demonstrated that a non-neuronal cholinergic system had a critical role in sustaining cellular ATP levels.

Furthermore, the actual ATP levels deduced by conventional luciferase activity measurements were well correlated with fold-increases in luciferase reporter activity. This suggested that this reporter method could be used instead of a cell-lysis-based ATP quantification method, because cell lysis is not required and ATP levels can be sequentially monitored by sampling the medium in which cells are incubated.

ChAT-expressing cells increased glucose uptake, because compared with control cells, 2-NDBG was more rapidly incorporated into ChAT-expressing cells. In addition, during the early phase of 2-NDBG uptake, signals appeared in a linear pattern along plasma membrane and even during the later phase, higher intensity signals were detected in the cytoplasm of ChAT-expressing cells. This appeared to be mediated by the non-hypoxic HIF-1 $\alpha$ induction pathway through $\mathrm{ACh}$, as per our previous study in which we reported that extrinsic ACh upregulated HIF-1 $\alpha$ protein expression through PI3K/Akt [5]. Therefore, we speculated that intrinsic ACh synthesized by ChAT-expressing cells also may have a role similar to that of extrinsic ACh. HIF-1 $\alpha$ is a master transcription factor responsible for glucose metabolism, including glycolysis and glucose uptake and utilization $[9,10,11]$. Thus, increased ACh levels in ChAT-expressing cells may have a crucial role in upregulating the HIF-1 $\alpha$ protein level and promote cells to use glucose more efficiently.

HEK293 cells subjected to transfection with reporter vectors was confirmed to express muscarinic and several nicotinic receptors based on results of Western blot and RT-PCR from our previous study [1]. Therefore, we speculated that cell-derived ACh, released into extracellular space, may exert its specific function through these receptors expressed by HEK293 cells in an autocrine and/or paracrine manner. Although direct evidence regarding a fashion for ACh release has not yet been obtained, some studies have indicated that synthesized ACh could be released into extracellular spaces through nonquantal mechanisms of secretion, i.e., a continuous release independent of ACh-containing vesicles [12, 13].

Based on the phenotypes induced by this activated non-neuronal cholinergic system, another advantage was verified in the present study. ChAT-expressing cells were resistant to prolonged serum deprivation (Fig. 6). The HEK293 cells often used in our studies were characterized under serum deprivation conditions. When cultured in DMEM alone, cell proliferation was suppressed, cell survival signals were attenuated, and increased cell death concomitant appearing round causing increased detachment from culture dishes had all occurred within 1 week. Compared with control cells, which usually died within 1 week of serum deprivation, more ChAT-expressing cells survived during that same period. Their survival rate was three times higher than that of control cells. The mechanism underlying this phenomenon was speculated to be that more ACh synthesized in ChAT-expressing cells increased glucose uptake by inducing glucose utilization-related gene expression by HIF$1 \alpha$, and this induction increased cellular ATP levels and sustained these ATP levels in the cytoplasm.

Till date, there have been no reports on the effects of ACh on cell survival under serum deprivation. However, results of other studies that used nicotine rather than ACh may agree 


\section{Cellular Physiology and Biochemistry}

Cell Physiol Biochem 2014;34:781-789

\begin{tabular}{l|l}
\hline DOI: $10.1159 / 000363042$ & (C) 2014 S. Karger AG, Basel
\end{tabular}

www.karger.com/cpb

Oikawa/Iketani/Kakinuma: A non-Neuronal Cholinergic System that Regulates Cellular ATP Levels

with the results of this study [14-16]. Furthermore, partly through a negative modulation of energy metabolism, as previously reported [1, 2], ATP consumption may be attenuated, which would contribute to upregulating ATP levels. Together with the present study results, we concluded that the activation of a non-neuronal cholinergic system resulted in ATP upregulation through increased utilization of glucose and negative regulation of ATP consumption.

\section{Acknowledgments}

This research was supported by The Vehicle Racing Commemorative Foundation and in part by a Grant-in-Aid from the Japan Society for the Promotion of Science (2540333). On behalf of all authors, the corresponding author states that there are no conflicts of interest to declare.

\section{References}

1 Kakinuma Y, Akiyama T, Sato T: Cholinoceptive and cholinergic properties of cardiomyocytes involving an amplification mechanism for vagal efferent effects in sparsely innervated ventricular myocardium. FEBS J 2009;276:5111-5125.

2 Kakinuma Y, Akiyama T, Okazaki K, Arikawa M, Noguchi T, Sato T: A non-neuronal cardiac cholinergic system plays a protective role in myocardium salvage during ischemic insults. PLoS One 2012;7:e50761.

-3 Kakinuma Y, Tsuda M, Okazaki K, Akiyama T, Arikawa M, Noguchi T, Sato T: Heart-specific overexpression of choline acetyltransferase gene protects murine heart against ischemia through hypoxia-inducible factor$1 \alpha$-related defense mechanisms. J Am Heart Assoc 2013;2:e004887.

-4 Patrizia Pellegatti, Simonetta Falzoni, Paolo Pinton, Rosario Rizzuto, Francesco Di Virgilio: Novel recombinant plasma membrane-targeted luciferase reveals a new pathway for ATP secretion. Mol Biol Cell 2005; 16:3659-3665.

-5 Kakinuma Y, Ando M, Kuwabara M, Katare RG, Okudela K, Kobayashi M, Sato T: Acetylcholine from vagal stimulation protects cardiomyocytes against ischemia and hypoxia involving additive non-hypoxic induction of HIF-1alpha. FEBS let 2005;579:2111-2118.

6 Kudo Y, Kakinuma Y, Mori Y, Morimoto N, Karashima T, Furihata M, Sato T, Shuin T, Sugiura T: Nephron Exp Nephrol 2005;100:e95-103.

7 Petty RD, Sutherland LA, Hunter EM, Cree IA: Comparison of MTT and ATP-based assays for the measurement of viable cell number. J Biolumin Chemilumin 1995;10:29-34.

8 Cree IA, Andreotti PE: Measurement of cytotoxicity by ATP-based luminescence assay in primary cell cultures and cell lines. Toxicol In Vitro 1997;11:553-536.

-9 Kurabayashi A, Kakinuma Y, Morita T, Inoue K, Sato T, Furihata M: Conditional VHL gene deletion causes hypoglycemic death associated with disproportionately increased glucose uptake by hepatocytes through an upregulated IGF-I receptor. PLoS One 2013;8:e69139.

10 Sakagami H, Makino Y, Mizumoto K, Isoe T, Takeda Y, Watanabe J, Fujita Y, Takiyama Y, Abiko A, Haneda M: Loss of HIF-1 $\alpha$ impairs GLUT4 translocation and glucose uptake by the skeletal muscle cells. Am J Physiol Endocrinol Metab 2014;306:E1065-E1076.

11 Xu H, Li B, Yu W, Wang H, Zhao X, Yao Y, Huang D: Correlation between ${ }^{18}$ F-FDG uptake and the expression of glucose transporter- 1 and hypoxia-inducible factor- $1 \alpha$ in transplanted VX2 tumors. Nucl Med Commun 2013;34:953-958.

12 Abramochkin DV, Borodinova AA, Rosenshtraukh LV, Nikolsky EE: Both neuronal and non-neuronal acetylcholine take part in non-quantal acetylcholine release in the rat atrium. Life Sci 2012;91:1023-1026.

-13 Borodinova AA, Abramochkin DV, Sukhova GS: Non-quantal release of acetylcholine in rat atrial myocardium is inhibited by noradrenaline. Exp Physiol 2013;98:1659-1667.

-14 Yamashita H, Nakamura S: Nicotine rescues PC12 cells from death induced by nerve growth factor deprivation. Neurosci Lett 1996;213:145-147.

-15 Tsurutani J, Castillo SS, Brognard J, Granville CA, Zhang C, Gills JJ, Sayyah J, Dennis PA: Tobacco components stimulate Akt-dependent proliferation and NFkappaB-dependent survival in lung cancer cells. Carcinogenesis 2005;26:1182-1195.

-16 Vatamaniuk MZ, Horyn OV, Vatamaniuk OK, Doliba NM: Acetylcholine affects rat liver metabolism via type 3 muscarinic receptors in hepatocytes. Life Sci 2003;72:1871-1882. 